\title{
Percutaneous Endoscopic Lumbar Discectomy for Huge Lumbar Disc Herniation with Complete Dural Sac Stenosis via an Interlaminar Approach: An Observational Retrospective Cohort Study
}

\author{
Cheng $\left.\mathrm{Ma} \mathbb{D}^{1}\right)^{1, *}$ \\ $\mathrm{He} \mathrm{Li}{ }^{1, *}$ \\ Yifan Wei ${ }^{I}$ \\ Lijia Liu' \\ Yin $\mathrm{Shi}^{2}$ \\ Yongxin Ren (D) \\ 'Department of Orthopaedics, The First \\ Affiliated Hospital of Nanjing Medical \\ University, Nanjing, Jiangsu, 210029 , \\ People's Republic of China; ${ }^{2}$ Department \\ of Radiology, The First Affiliated Hospital \\ of Nanjing Medical University, Nanjing, \\ Jiangsu, 21 0029, People's Republic of \\ China \\ *These authors contributed equally to \\ this work
}

\begin{abstract}
Purpose: Percutaneous endoscopic lumbar discectomy (PELD) is a minimally invasive spinal surgery for huge lumbar disc herniation (HLDH). The aim of this study was to investigate the short-term clinical effectiveness of PELD for HLDH with complete dural sac stenosis via an interlaminar approach.

Methods: We retrospectively analyzed 56 patients diagnosed with HLDH with complete dural sac stenosis and treated with PELD via an interlaminar approach. Numerical rating scale (NRS), Oswestry disability index (ODI), and modified Japanese orthopedic association (mJOA) were used to evaluate preoperative conditions as well as outcomes at 1, 3, 6 and 12 months postoperatively. At the final follow-up, the clinical effects were evaluated using modified MacNab criteria.

Results: All patients were followed for at least 12 months. At 1, 3, 6, and 12 months postoperatively, the NRS and ODI scores were significantly decreased, and the mJOA score significantly increased compared with preoperative results $(\mathrm{P}<0.001)$. According to the Macnab criteria at the final follow-up, it was excellent in 42 patients (75\%), good in 9 (16.1\%), and fair in 5 (8.9\%). The overall clinical satisfactory rate was $91.1 \%$.

Conclusion: Our study results suggest that percutaneous endoscopic interlaminar discectomy (PEID) is available for the treatment of HLDH with complete dural sac stenosis, whose benefits are rapid recovery, complete removal of the herniated disc, effective spinal canal decompression, fewer complications, and significant relief of clinical symptoms.

Keywords: percutaneous endoscopic lumbar discectomy, percutaneous endoscopic interlaminar discectomy, huge lumbar disc herniation, complete dural sac stenosis, diffusion tensor imaging
\end{abstract}

\section{Introduction}

Percutaneous endoscopic lumbar discectomy (PELD) is a minimally invasive spinal surgery for $\mathrm{LDH}$ that has rapidly evolved and gained popularity over recent years. ${ }^{1}$ Compared to traditional posterior open lumbar discectomy or minimally invasive surgical techniques, the advantages of PELD such as less paravertebral muscle injury and blood loss, greater preservation of the bony structure, shorter hospital stay and earlier return to work have been widely accepted by many spinal surgeons. ${ }^{2}$ PELD can be mainly divided into two different approaches: the interlaminar approach (PEID) and the transforaminal approach (PETD). ${ }^{3}$ Since
Correspondence: Yongxin Ren; Yin Shi Tel +86 I3585I62283

Email renyongxinjsph@163.com; shiyin_ njmu@foxmail.com 
Professor Ruetten proposed PEID in 2006, it has been widely accepted by surgeons because it avoids the restrictions of high iliac crest and transverse process, the visual field similar to open surgery, and more direct intra-spinal decompression. ${ }^{4,5}$ With the update of instruments and the development of technology, the indications of PELD are also expanding accordingly, such as recurrent $\mathrm{LDH}$ and calcified LDH, which have been reported in many literatures that can be treated by PELD. ${ }^{6}$

Huge lumbar disc herniation (HLDH) is a special type of lumbar disc herniation, generally defined as herniation that occludes more than $50 \%$ area of the spinal canal and impinges on neural structures on computed tomography (CT) scans and magnetic resonance images (MRI). Compared to general LDH, HLDH usually results in more severe back and leg pain, as well as higher incidence of Cauda equina syndrome (CES). ${ }^{7,8}$ Among these patients with $\mathrm{HLDH}$, while no recognizable nerve root and cerebrospinal fluid signal on T2-weighted axial MRI was observed, it was defined as complete dural sac stenosis. This often attracts clinicians and patients' concern, who preferred the operative treatment due to fear of CES or significant neurological dysfunction. ${ }^{8,9}$

Yet, to date, few data are available on the clinical effectiveness of PEID for HLDH with complete dural sac stenosis. In this retrospective study, we investigated the preliminary results in $56 \mathrm{HLDH}$ patients with complete dural sac stenosis treated with PELD via an interlaminar approach.

\section{Materials and Methods}

\section{Patients}

A total of 56 patients who were diagnosed with HLDH with complete dural sac stenosis based on the clinical manifestations, CT, MRI and diffusion tensor imaging (DTI) between February 2017 and December 2019 were enrolled in the study. All the patients were treated with PELD via an interlaminar approach by a single surgeon at the orthopedics department, the first affiliated hospital of Nanjing Medical University, Nanjing, China. The inclusion criteria included: (1) patients diagnosed with HLDH with complete dural sac stenosis; (2) patients complaining of low back and lower limb pain or numbness and motor weakness due to huge lumbar disc herniation; (3) symptoms corresponding to preoperative MRI and CT scans; (4) failure of 6-week conservative treatment. The exclusion criteria were as follows: (1) patients with organ dysfunction or hemorrhagic tendency; (2) vertebral infection or tumor; (3) Lumbar spondylolisthesis or degenerative deformities, instability, and scoliosis. The study was conducted in accordance with the Helsinki Declaration and approved by the Institutional Review Board of the first affiliated hospital of Nanjing medical university. Written informed consent was obtained from all patients or their legal guardian, allowing their clinical data to be further analyzed.

\section{Surgical Technique}

The PEID was performed under general anesthesia with the patient in a prone position on a $\mathrm{C}$-arm fluororadiolucent table A pillow was placed between the lower abdomen and chest to permit the free suspension of the abdomen. The target level and the skin entry point were confirmed by using anterior-posterior (AP) fluoroscopy, and the puncture needle was inserted from the marked skin entry point to the lateral margin of the interlaminar window, after which the needle was replaced with an $18 \mathrm{G}$ guidewire (Figure $1 \mathrm{~A}$ and B). A tapered cannulated obturator was then passed over the guidewire until its tip reached the superior border of the lower vertebral lamina, as observed in the AP view. Subsequently, a working cannula was passed over the obturator (Figure 1C). When the position was corrected according to fluoroscopic guidance (Figure 1D and E), the endoscope was positioned after the obturator and guidewire were removed, and the procedure was continued under direct visualization with normal saline irrigation maintained at a constant rate. Rotate the sheath to push away the surrounding muscle tissue, then the ligamentum flavum and facet joints will be exposed. Similar to open surgery, we first removed part of the lamina and the medial edge of the facet joint with trephine and Kerrison rongeur to complete bone decompression (the range is larger than that of conventional PEID). After breaking the ligamentum flavum with punch forceps, remove the ligamentum flavum and expose the edge of the dural sac and the nerve root with Kerrison rongeur. After this step, the dorsal decompression of the dural sac was basically completed. This is to reserve enough space for the dural sac when the working cannula enters the spinal canal to avoid further damage to the dural sac. The huge herniated disc was segmented and removed with nucleus pulposus forceps through the nerve root shoulder or nerve root axillary (Figure 1H). After complete decompression, the dural sac and lumbar-exiting nerve root were carefully checked for being freely movable (Figure 1I). Finally, the working cannula was carefully removed, and skin was closed with a single suture (Figure 1J and K). 

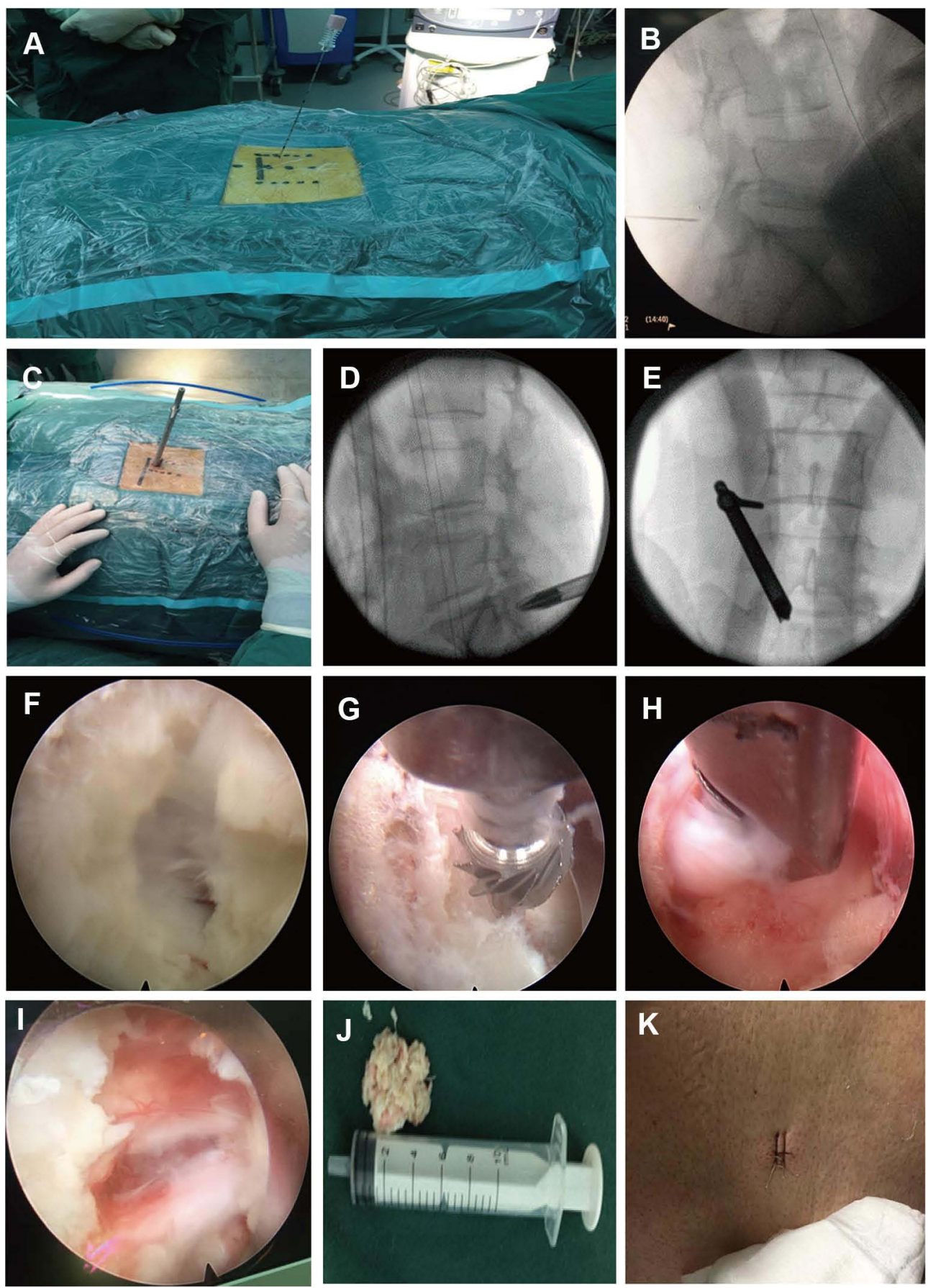

Figure I Surgical technique. (A) A puncture needle was inserted from the marked skin entry point to the lateral margin of the interlaminar window. (B) Intraoperative lateral fluoroscopic images showing a puncture needle located at the interlaminar window of the L5/SI level. (C) A working cannula was placed through the obturator from the marked skin entry point to the lateral margin of the interlaminar window. (D and E) Intraoperative AP and lateral fluoroscopic images showing the placement of the working cannula. (F) Incising the ligamentum flavum sequentially using nucleus forceps. (G) Bony resection of inner margin of the superior articular process using a highspeed endoscopic drill system. (H) Removing the intracanal nucleus pulposus by using nucleus forceps. (I) Free movement of SI nerve root after removal of the herniation. (J) Sequestrated disc pulposus fragments. (K) Operation incision.

\section{Observation Parameters}

All the patients were followed-up by telephone or outpatient interviews for 13 to 15 months. Clinical data and perioperative parameters were collected. Patients received lumbar MRI and DTI at 3 months postoperatively to evaluate the resection completeness of disc pulposus fragments (Figure 2). During the hospital stay and the follow-up period, complications such as iatrogenic nerve damage were recorded. Numerical rating scale (NRS), modified Japanese Orthopedic Association (mJOA) score, and ODI (Oswestry 

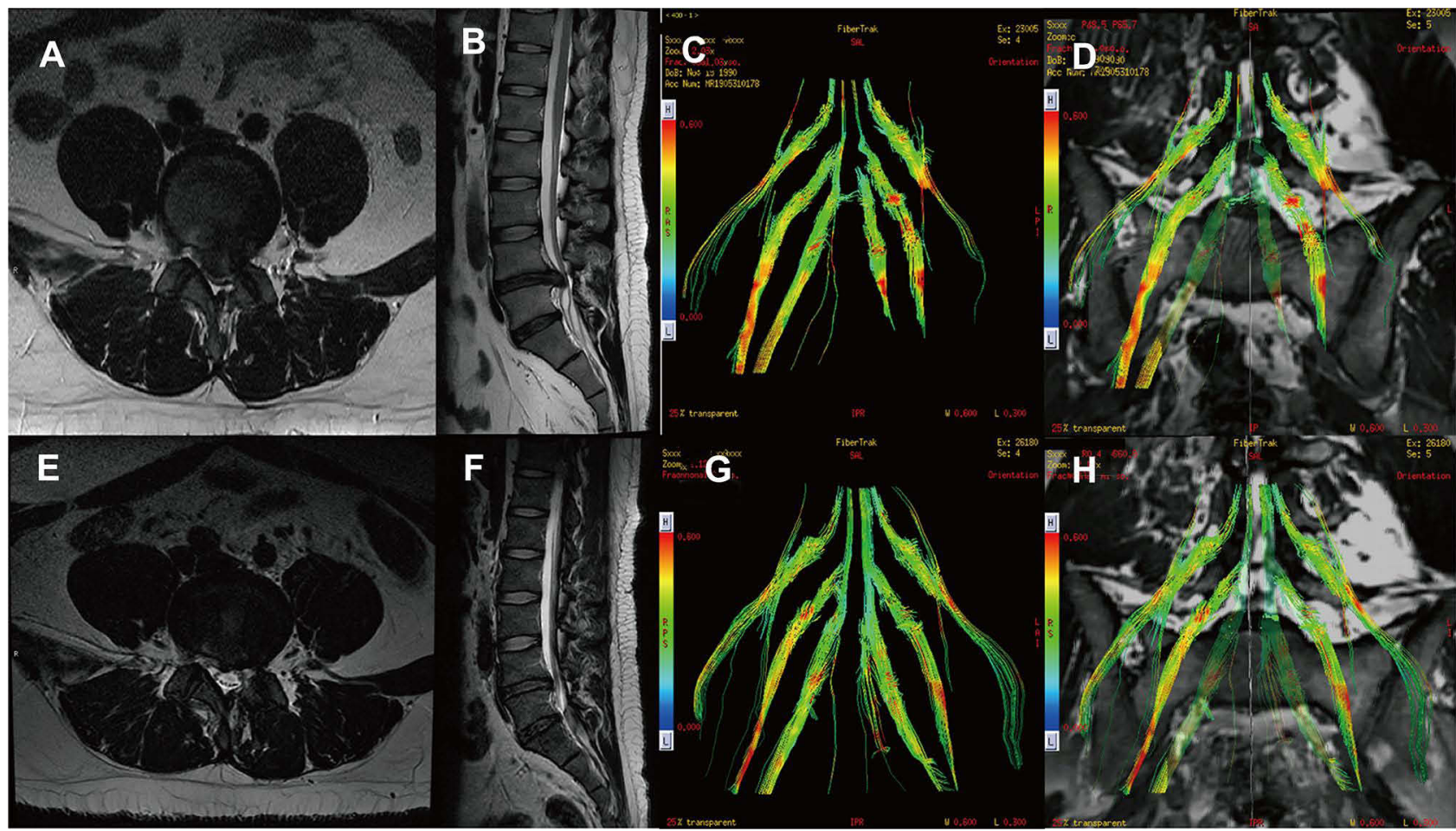

Figure 2 A 28-year-old woman had been suffering from radiating pain in the left leg for seven months. Preoperative sagittal (A) and axial (B) MRI showing huge disc herniation with complete dural sac stenosis at the L4/5 level. Diffusion tensor tractography (C and D), with colored FA scale fiber tracking, distinctly showed abnormalities located at the left L5 and SI nerve root. Postoperative sagittal (E) and axial (F) MRI shows a large annular defect and partial removal of the nucleus pulposus and the close contact between the annulus fibrosus and the nerve root. Diffusion tensor tractography $(\mathbf{G}$ and $\mathbf{H})$ with colored FA scale fiber tracking showed no obvious compression of the left 5 and SI nerve root. Preoperation vs postoperative 3 months, ODI: 57.78 vs 20; NRS: 7 vs 2; mJOA: 8 vs 16 .

Disability Index Questionnaire) were applied to evaluate the preoperative condition as well as the curative effectiveness at 1, 3, 6 and 12 months, postoperatively. Modified Macnab criteria were applied at the final follow-up. ${ }^{10}$ Excellent and good rates were defined as clinically satisfactory.

\section{Statistical Analysis}

The results were presented as mean \pm standard deviation (SD) or median and interquartile range (IQR). Paired $t$-test or Wilcoxon test was used to compare the differences preand post-operation. The GraphPad Prism (version 8.0.0, San Diego, California USA) was used for statistical analyses, and results were considered significant at $\mathrm{P}<0.05$.

\section{Results}

\section{Sociodemographic and Clinical Characteristics of the Patients}

There were 56 ( 35 males and 21 females) patients with ages ranging from 17 to 75 years (mean $44.45 \pm 13.41$ years). They suffered from various degrees of unilateral or bilateral lower limb pain and low back pain, some of whom presented with paresthesia, dermatomal hypesthesia, or weakness in the region of the affected nerve root and/or positive straight leg raising, according to a physical examination. The median duration of symptoms was 30.5 months (range, 2-123 months). Only 1 patient presented CES. Their preoperative MRI, DTI and CT scans were in compliance with clinical findings. Infected levels were L4/5 in 20 patients and L5/S1 in 36. The duration of the surgery ranged from 60 to 150 (mean 101 \pm 23 ) minutes. The hospital stay ranged from 2 to 7 (mean $3.9 \pm 1.5$ ) days (Table 1).

\section{Clinical Outcomes}

The median value of NRS for back and leg pain at preoperation and postoperative 1, 3, 6, and 12 months was 7 (range, 6-9), 3 (range, 2-4), 2 (range, 1-3), 2 (range, 1-2) and 1 (range, $0-2$ ), respectively. The median value of mJOA score at preoperation and postoperative 1, 3, 6, and 12 months were 8 (range, 6-11), 16 (range, 14-19), 16 (range, 15-19), 18 (range, 15-19) and 18 (range, 16$19)$, respectively. The median value of ODI score at preoperation and postoperative 1, 3, 6, and 12 months were 54.45 (range, 40-62.22), 20 (range, 15.56-24.44), 17.78 (range, 15.56-20), 17.78 (range, 13.33-20) and 15.56 
Table I Sociodemographic, Clinical Characteristics of the Patients

\begin{tabular}{|l|c|c|}
\hline No. of Patients & & 56 \\
\hline Age (years) & & $44.45 \pm|3.4|$ \\
\hline Sex (M/F) & & $35 / 2 I$ \\
\hline Low back pain & Unilateral & 30 \\
\hline Leg pain & Bilateral & 60 \\
\hline Disc level & L4/5 & 20 \\
& L5/SI & 36 \\
\hline Paresthesia in lower leg & & 47 \\
\hline Muscle weakness & EHL & 7 \\
& $\mathrm{FHL}$ & 6 \\
\hline Dermatomal hypesthesia & & 44 \\
\hline Symptom duration (months) & & $30.5(9-52.75)$ \\
\hline Lasegue test (+) & & 48 \\
\hline Operative time (min) & & $101 \pm 23$ \\
\hline Hospital stay (days) & & $3.9 \pm 1.5$ \\
\hline
\end{tabular}

Abbreviations: $\mathrm{M}$, male; F, female; +, presence; -, absence; FHL, flexor hallucis longus; EHL, extensor hallucis longus.

(range, 13.33-17.78), respectively. The NRS and ODI scores at 1, 3, 6, 12 months postoperatively showed a significant decrease compared to preoperative results $(\mathrm{P}<0.01)$. The mJOA score at $1,3,6,12$ months postoperatively was significantly higher than the preoperative condition $(\mathrm{P}<0.001)$ (Figure 3$)$. Macnab criteria at the final follow-up were excellent in 42 patients $(75 \%)$, good in $9(16.1 \%)$, and fair in $5(8.9 \%)$. The overall clinically satisfactory rate was $91.1 \%$ (Table 2). Disc infection, permanent iatrogenic nerve damage, dural tear, cerebrospinal fluid leakage, intraoperative vascular injury, recurrent disc herniation, and other significant complications were not observed. One patient complained of postoperative temporary lower limb hyperalgesia. The symptoms indicated improvement 2 months postoperatively after conservative treatment. One patient suffered from bilateral lower limb pain, which was more severe than before the operation. The signs were gone within 3 days following an intravenous infusion of flurbiprofen axetil.

\section{Discussion}

LDH is the leading cause of CES. While HLDH with complete dural sac stenosis is more likely to be associated with CES, not every patient diagnosed with HLDH with complete dural sac stenosis will present CES. ${ }^{7,8}$ In our study, only one patient abruptly presented with bladder dysfunction and muscular weakness in legs after sneezing, which suggested that the rate of CES was not severely affected by complete obliteration of the dural sac, which was consistent with previous studies. ${ }^{11,12}$ The patient underwent surgery within 24 hours of onset. The symptoms of bladder dysfunction and bilateral paresis completely disappeared after five days. For decades, minimally invasive technology has been widely adopted in various disciplines to compensate for the disadvantages of traditional open surgery. Since Kambin et al firstly reported that the patients with clinical and myelographic evidence of disc herniation treated with percutaneous lateral discectomy had good outcomes in $1986,{ }^{13}$ PELD has undergone rapid development and significant innovation, gradually evolving as the optimal choice for LDH. ${ }^{14}$ However, the choice of surgical procedure for HLDH remains controversial. Traditionally, due to the removal of protruding and degenerative nucleus pulposus fragments in relieving nerve compression, which could increase the risk of postoperative spinal instability and chronic back pain, ${ }^{15-17}$ Zhao et al suggested TLIF as an effective treatment option for HLDH. ${ }^{18}$ Satoh et al indicated that PLIF could be used as a surgical treatment for lumbar disc herniation with the massive herniation or segmental instability. ${ }^{19}$ With the update of instruments and the development of technology, the indications of PELD are also expanding accordingly. As a result, a growing number of studies on PELD for HLDH and the comparison between PELD and conventional lumbar discectomy have been reported over the recent years. ${ }^{20,21}$ Kondo et al reported on 11 consecutive patients with large central LDH who underwent PELD with good clinical outcomes. ${ }^{22}$ We also obtained the same results in this study.

In the present study, we focused on the efficacy of PEID in HLDH patients with complete dural sac stenosis, with a special interest in the improvement of sensory and motor functions. We observed that the NRS and ODI scores rapidly decreased, while mJOA scores quickly increased postoperatively, thus indicating a significant relief of clinic symptoms. According to the MacNab criteria, the overall excellent and good rate was $91.1 \%$, which suggested satisfactory clinical outcomes. Significant complications, such as permanent iatrogenic nerve damage, dural tear, and cerebrospinal fluid leakage, were not 
A
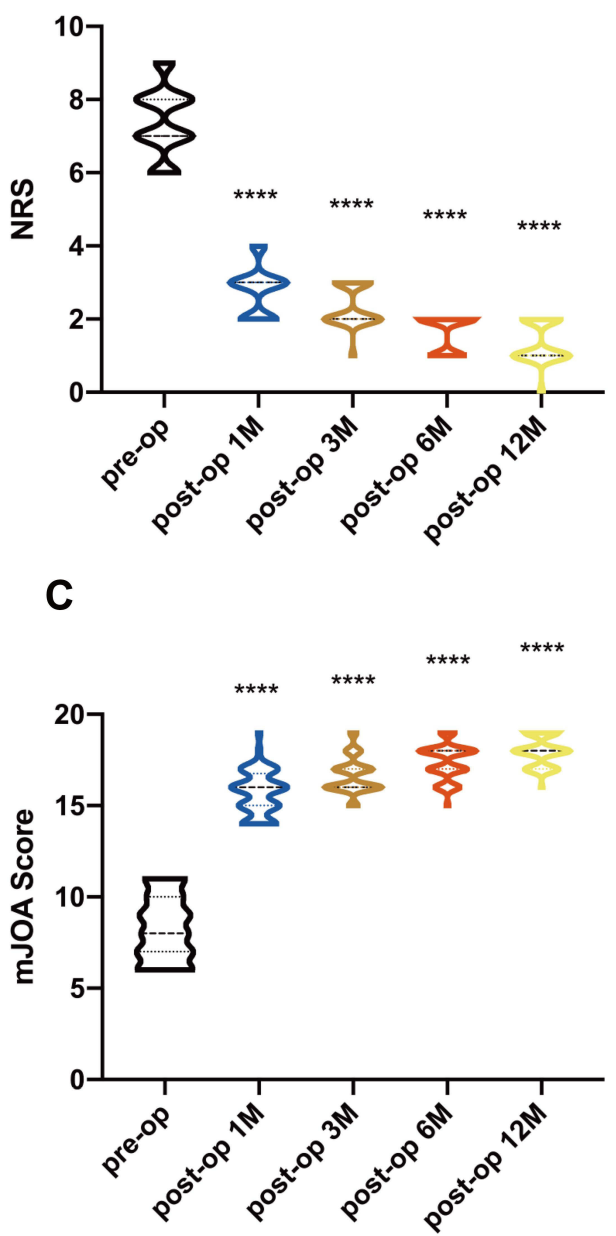

B

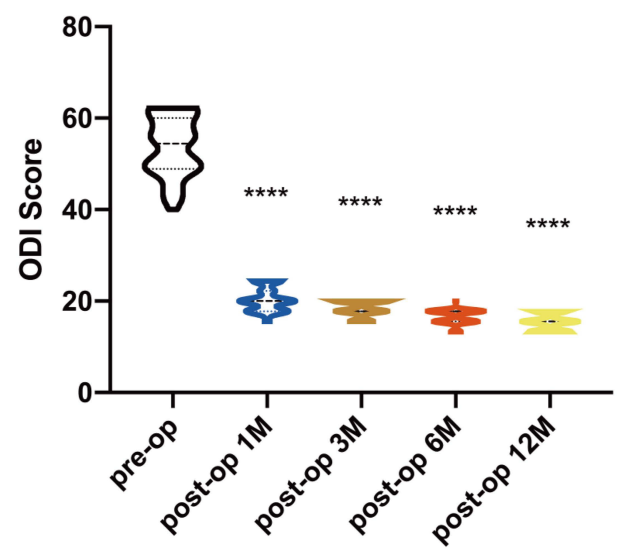

Figure 3 Violin plots of pre- and postoperative I, 3, 6, and I2 months NRS, mJOA and ODI scores (A-C). The NRS and ODI scores at I, 3, 6, 12 months postoperatively showed a significant decrease compared to preoperative results $(\mathrm{P}<0.01)$. The $\mathrm{mJOA}$ score at $\mathrm{I}, 3,6,12$ months postoperatively was significantly higher than the preoperative condition. (****p $<0.0001$ ).

observed. Yet, pain is relieved after conservative treatment in 2 patients who experienced transient lower limb hyperalgesia and bilateral lower limb pain, which was relative to the compression or irritation of the nerve root. Sensory

Table 2 MacNab Criteria

\begin{tabular}{|l|c|c|c|}
\hline Outcomes & Frequency & $\%$ & Cumulative \% \\
\hline Excellent & 42 & 75 & 75 \\
Good & 9 & 16.1 & 91.1 \\
Fair & 5 & 8.9 & 100 \\
Poor & 0 & 0 & 100 \\
Total & 56 & 100 & - \\
\hline
\end{tabular}

Abbreviations: PELD, percutaneous endoscopic lumbar discectomy; HLDH, huge lumbar disc herniation; CES, Cauda equina syndrome; DTI, diffusion tensor imaging; FA, fractional anisotropy; MRI, magnetic resonance imaging; CT, computed tomography. disturbance, muscle weakness, low back, and leg pain were alleviated to different degrees after the operation. These results revealed that PEID was an effective option for treating HLDH with complete dural sac stenosis.

Moreover, the huge herniation often leads to more serious low back and leg pain, and is more prone to multiple lumbosacral segment and bilateral nerve root compression in radiographic imaging. Conventional MRI is an effective imaging method for the diagnosis of lumbosacral radiculopathy, especially for lumbar disc herniation associated with radiculopathy. However, the complexity of huge herniated intervertebral disc compressed nerves may limit its further application in the evaluation of lumbosacral radiculopathy in HDLH, which is not conducive to the location of the responsible segment at preoperation and the evaluation of postoperative curative effect. 
Therefore, diffusion tensor imaging (DTI), as an advanced magnetic resonance imaging technology, has been applied in this study. Shi et al have shown that the DTI derived fractional anisotropy (FA) values can be a potential marker for quantitative and clinical evaluation of nerve root in lumbosacral radiculopathy. ${ }^{23}$ Interestingly, although the herniated intervertebral disc that has been removed can be observed on conventional MRI at postoperative 3 months, the close contact between the annulus fibrosus and the nerve root can still be seen in some patients whose pain symptoms are significantly relieved, and the nerve function is obviously improving. However, in our study, we used DTI fiber tracking, which can allow for 3D insights into the normal anatomy and general organization of the lumbosacral plexus and its branching nerves to directly show the location of compressed nerve root at preoperation and directly reflect the nerve recovery postoperatively, which is beneficial for clinical assessment. Additionally, based on DTI FA parameters, diffusion tensor tractography can also locate the compressed nerve roots to further clarify the responsible intervertebral disc at preoperatively, assisting the surgeon to perform precise surgery. We recommend that DTI be included in the routine imaging examination of HLDH.

Although surgery for the treatment of HLDH with complete dural sac stenosis is riskier and more challenging than the general lumbar disc herniation, such as intraoperative neural injury, dural tear, artery injury, and cerebrospinal fluid leakage, there were no major complications observed in this study. The following points need to be carefully considered during surgery. Firstly, the ligamentum flavum should be excised sequentially using Kerrison rongeur under visual control, after which the tunnel should be enlarged so as to insert a working cannula. Compared to the linear splitting of the ligamentum flavum with the dissector, this method is safer; however, the injury to the ligamentum flavum cannot be prevented, which may cause instability and scarring of the epidural space. Secondly, when the dural sac and nerve root are compressed by a huge disc, surgeons cannot always identify them clearly. Part of the herniated fragment was grasped to decompress the spinal canal and expand the working space and field of vision. The working cannula cannot enter the spinal canal before the margin of the dural sac and nerve root were clearly detected. Thirdly, the extensive resection of ligamentum flavum and bony resection of the inner margin of the superior articular process by using a high-speed endoscopic drill system might be necessary for decompressing the spinal canal. Furthermore, to avoid excessive extruding nerve root oppressed by HLDH causing iatrogenic injury when the working cannula is rotating, it is recommended to segmented remove the herniated nucleus pulposus from the spinal canal first. After complete removal of the detectable nucleus pulposus, the involved nerve root needs to be carefully examined under endoscopy to ascertain its mobility. Finally, the learning curve of PEID for surgeons is relatively flat and hard to master. ${ }^{24}$ Surgeons need to convert the 2-dimensional vision of the endoscope on the monitor into the 3-dimensional anatomical model in mind. Gaining adequate anatomy knowledge, training on the cadaver, and performing the procedure under expert supervision in at least the initial 10 cases are essential for preventing intraoperative complications. ${ }^{25}$

This retrospective study has several limitations. There was no control group, which made it difficult to determine whether PEID was superior compared to traditional open surgery. The sample size is relatively small, which suggests the possibility of clinical heterogeneity. In addition, the duration of follow-up was short, and only a few observational indicators were used. Patients were not taken MRI and DTI scanning after three months postoperatively for assessment. Future large sample, randomized controlled, and long-term follow-up studies are needed to further verify our findings.

\section{Conclusion}

The findings of our study suggest that PEID may be an effective option for the treatment of HLDH with complete dural sac stenosis. In the future, prospective randomized controlled trials with larger sample sizes are needed to verify the clinical efficacy of PEID in the treatment of HLDH.

\section{Acknowledgments}

Special thanks to Professor Dengshun Miao; Dr. Hui Che; Dr. You Li, Dr. Huan Liu, and Dr. Yimin Li for all their help. Cheng $\mathrm{Ma}$ and $\mathrm{He} \mathrm{Li}$ are co-first authors of this study.

\section{Disclosure}

The authors report no conflicts of interest in this work.

\section{References}

1. Chen J, Jing X, Li C, Jiang Y, Cheng S, Ma J. Percutaneous endoscopic lumbar discectomy for L5S1 lumbar disc herniation using a transforaminal approach versus an interlaminar approach: a systematic review and meta-analysis. World Neurosurg. 2018;116:412-20 e2. doi:10.1016/j.wneu.2018.05.075 
2. Ruan W, Feng F, Liu Z, Xie J, Cai L, Ping A. Comparison of percutaneous endoscopic lumbar discectomy versus open lumbar microdiscectomy for lumbar disc herniation: a meta-analysis. Int J Surg. 2016;31:86-92. doi:10.1016/j.ijsu.2016.05.061

3. Wang D, Xie W, Cao W, He S, Fan G, Zhang H. A cost-utility analysis of percutaneous endoscopic lumbar discectomy for L5-S1 lumbar disc herniation: transforaminal versus interlaminar. Spine (Phila Pa 1976). 2019;44(8):563-570. doi:10.1097/BRS.0000000 000002901

4. Ruetten S, Komp M, Godolias G. A new full-endoscopic technique for the interlaminar operation of lumbar disc herniations using 6-mm endoscopes: prospective 2-year results of 331 patients. Minim Invasive Neurosurg. 2006;49(2):80-87. doi:10.1055/s-2006-932172

5. Kim CH, Chung CK, Sohn S, Lee S, Park SB. The surgical outcome and the surgical strategy of percutaneous endoscopic discectomy for recurrent disk herniation. J Spinal Disord Tech. 2014;27(8):415-422. doi:10.1097/BSD.0b013e3182a180fc

6. Dabo X, Ziqiang C, Yinchuan Z, et al. The clinical results of percutaneous endoscopic interlaminar discectomy (PEID) in the treatment of calcified lumbar disc herniation: a case-control study. Pain Physician. 2016;19(2):69-76. doi:10.36076/ppj/2016.19.69

7. Akhaddar A, Belfquih H, Salami M, Boucetta M. Surgical management of giant lumbar disc herniation: analysis of 154 patients over a decade. Neurochirurgie. 2014;60(5):244-248. doi:10.1016/j.neuchi. 2014.02.012

8. Jeon $\mathrm{CH}$, Chung NS, Son KH, Lee HS. Massive lumbar disc herniation with complete dural sac stenosis. Indian J Orthop. 2013;47 (3):244-249. doi:10.4103/0019-5413.111505

9. Foruria X, Ruiz de Gopegui K, Garcia-Sanchez I, Moreta J, Aguirre U, Martinez-de Los Mozos JL. Cauda equina syndrome secondary to lumbar disc herniation: surgical delay and its relationship with prognosis. Rev Esp Cir Ortop Traumatol. 2016;60 (3):153-159. doi:10.1016/j.recot.2016.01.003

10. Macnab I. Negative disc exploration. An analysis of the causes of nerve-root involvement in sixty-eight patients. J Bone Joint Surg Am. 1971;53(5):891-903. doi:10.2106/00004623-197153050-00004

11. Bernhardt M, Gurganious LR, Bloom DL, White AA. Magnetic resonance imaging analysis of percutaneous discectomy. A preliminary report. Spine (Phila Pa 1976). 1993;18(2):211-217. doi:10.1097/00007632-199302000-00007

12. Cribb GL, Jaffray DC, Cassar-Pullicino VN. Observations on the natural history of massive lumbar disc herniation. $J$ Bone Joint Surg Br. 2007;89(6):782-784. doi:10.1302/0301-620X.89B6.18712

13. Kambin P, Sampson S. Posterolateral percutaneous suction-excision of herniated lumbar intervertebral discs. Report of interim results. Clin Orthop Relat Res. 1986;207:37-43.
14. Kim M, Lee S, Kim HS, Park S, Shim SY, Lim DJ. A comparison of percutaneous endoscopic lumbar discectomy and open lumbar microdiscectomy for lumbar disc herniation in the Korean: a meta-analysis. Biomed Res Int. 2018;2018:9073460. doi:10.1155/2018/9073460

15. Yorimitsu E, Chiba K, Toyama Y, Hirabayashi K. Long-term outcomes of standard discectomy for lumbar disc herniation: a follow-up study of more than 10 years. Spine (Phila Pa 1976). 2001;26 (6):652-657. doi:10.1097/00007632-200103150-00019

16. Maroon JC, Abla A, Bost J. Association between peridural scar and persistent low back pain after lumbar discectomy. Neurol Res. 1999;21(Suppl 1):S43-S46. doi:10.1080/01616412.1999.11741026

17. Iatridis JC, Ap Gwynn I. Mechanisms for mechanical damage in the intervertebral disc annulus fibrosus. $J$ Biomech. 2004;37 (8):1165-1175. doi:10.1016/j.jbiomech.2003.12.026

18. Zhao CQ, Ding W, Zhang K, Zhao J. Transforaminal lumbar interbody fusion using one diagonal fusion cage with unilateral pedicle screw fixation for treatment of massive lumbar disc herniation. Indian J Orthop. 2016;50(5):473-478. doi:10.4103/0019-5413.189595

19. Satoh I, Yonenobu K, Hosono N, Ohwada T, Fuji T, Yoshikawa H. Indication of posterior lumbar interbody fusion for lumbar disc herniation. J Spinal Disord Tech. 2006;19(2):104-108. doi:10.1097/ 01.bsd.0000180991.98751.95

20. Choi KC, Kim JS, Ryu KS, Kang BU, Ahn Y, Lee SH. Percutaneous endoscopic lumbar discectomy for L5-S1 disc herniation: transforaminal versus interlaminar approach. Pain Physician. 2013;16(6):547-556.

21. Liu C, Zhou Y. Percutaneous endoscopic lumbar discectomy and minimally invasive transforaminal lumbar interbody fusion for massive lumbar disc herniation. Clin Neurol Neurosurg. 2019;176:19-24. doi:10.1016/j.clineuro.2018.10.017

22. Kondo M, Oshima Y, Inoue H, Takano Y, Inanami H, Koga H. Significance and pitfalls of percutaneous endoscopic lumbar discectomy for large central lumbar disc herniation. J Spine Surg. 2018;4 (1):79-85. doi:10.21037/jss.2018.03.06

23. Shi Y, Zou Y, Feng Y, et al. A quantitative and clinical evaluation of nerve roots in lumbosacral radiculopathy using diffusion tensor imaging. Jpn J Radiol. 2020;38(3):222-230. doi:10.1007/s11604019-00913-4

24. Hsu HT, Chang SJ, Yang SS, Chai CL. Learning curve of full-endoscopic lumbar discectomy. Eur Spine J. 2013;22 (4):727-733. doi:10.1007/s00586-012-2540-4

25. Choi G, Lee SH, Raiturker PP, Lee S, Chae YS. Percutaneous endoscopic interlaminar discectomy for intracanalicular disc herniations at L5-S1 using a rigid working channel endoscope. Neurosurgery. 2006;58 (1Suppl):SONS59-SONS68. doi:10.1227/01.neu.0000192713.95921.4a
International Journal of General Medicine

\section{Publish your work in this journal}

The International Journal of General Medicine is an international, peer-reviewed open-access journal that focuses on general and internal medicine, pathogenesis, epidemiology, diagnosis, monitoring and treatment protocols. The journal is characterized by the rapid reporting of reviews, original research and clinical studies across all disease areas. The manuscript management system is completely online and includes a very quick and fair peer-review system, which is all easy to use. Visit http://www.dovepress.com/ testimonials.php to read real quotes from published authors. 